Journal for ImmunoTherapy of Cancer

\title{
Combined DLL3-targeted bispecific antibody with PD-1 inhibition is efficient to suppress small cell lung cancer growth
}

\author{
Xin Chen, ${ }^{1}$ Norhan Amar (D) , ${ }^{1}$ Yuankui Zhu, ${ }^{1}$ Chunguang Wang, ${ }^{2}$ Chunjiao Xia, ${ }^{1}$ \\ Xiaoqing Yang, ${ }^{3}$ Dongde Wu, ${ }^{4}$ Mingqian Feng (i) ${ }^{1}$
}

To cite: Chen X, Amar N, Zhu Y, et al. Combined DLL3-targeted bispecific antibody with PD-1 inhibition is efficient to suppress small cell lung cancer growth. Journal for ImmunoTherapy of Cancer 2020;8:e000785. doi:10.1136/jitc-2020-000785

Accepted 11 May 2020

\section{Check for updates}

C Author(s) (or their employer(s)) 2020. Re-use permitted under CC BY-NC. No commercial re-use. See rights and permissions. Published by BMJ.

${ }^{1}$ College of Life Science and Technology, Huazhong Agricultural University, Wuhan, Hubei, China

${ }^{2}$ Department of Thoracic Surgery, Jilin University Second Hospital, Changchun, Jilin, China ${ }^{3} \mathrm{Hospital}$ of Huazhong Agricultural University, Huazhong Agricultural University, Wuhan, Hubei, China

${ }^{4}$ Department of Hepatobiliary and Pancreatic Surgery, Hubei Cancer Hospital, Wuhan, Hubei, China

\section{Correspondence to} Dr Mingqian Feng; fengmingqian@mail.hzau. edu.cn

\section{ABSTRACT}

Background Small cell lung cancer (SCLC) accounts for $15 \%$ of lung cancers, and the primary treatment of this malignancy is chemotherapy and radiotherapy. Delta-like 3 (DLL3) is an attractive target for SCLC immunotherapy since its expression is highly restricted to SCLC with a neglectable appearance on normal adult tissues. In the current study, we aimed to explore the efficacy of DLL3targeted SCLC immunotherapy via the engagement of T cell.

Methods As a proof of concept, we constructed DLL3targeted bispecific antibody and chimeric antigen receptor (CAR)-modified T cells. In vitro and in vivo tumor-suppression activity of these treatments alone or in combination with a Program Death-1 (PD-1) inhibitory antibody was evaluated.

Results In vitro studies showed that both DLL3 bispecific antibody and CAR-T efficiently killed DLL3-positive cancer cells, including the native SCLC cell lines H446, H196, $\mathrm{H} 82$, and the artificial A431 cells that were forcefully overexpressing DLL3. In vivo studies in xenograft mouse models demonstrated that both bispecific antibody and CAR-T suppressed the tumor growth, and combination therapy with PD-1 inhibitory antibody dramatically improved the efficacy of the DLL3 bispecific antibody, but not the CAR-T cells.

Conclusions Our results demonstrated that DLL3targeted bispecific antibody plus PD-1 inhibition was effective in controlling SCLC growth.

\section{BACKGROUND}

Small cell lung cancer (SCLC) is a subtype of lung cancer, which accounts for about $15 \%$ of all lung cancer cases. ${ }^{1-3}$ SCLC is notorious for its high degree of malignant attributes, including fast growth, early tendency to widespread metastasis, and poor prognosis. ${ }^{1-3}$ According to the staging system of the Veterans Administration Lung Study Group, SCLC can be classified as the limited disease (early stage) and extensive disease (late or advanced stage). ${ }^{4}$ Approximately $70 \%$ of patients are diagnosed at extensive stage, which is frequently accompanied with distant metastasis. ${ }^{12}$

By far, systemic chemotherapy and localized radiotherapy still represent the main treatment modalities for SCLC. According to the international standard of care, patients with SCLC with extensive stage and widespread metastasis are typically treated with chemotherapy cisplatin or carboplatin together with etoposide. ${ }^{5}$ For early stage of SCLC, local radiotherapy combined with chemotherapy is recommended. Although SCLC is sensitive to the initial chemotherapy and radiotherapy, it has a very high rate of relapse and drug tolerance. ${ }^{2}$

In recent years, immunotherapy has achieved great clinical success in non-SCLC and other cancer types. However, the progress on SCLC immunotherapy is far from satisfaction, and the mainstay of clinical trials in SCLC immunotherapy is restricted to immune checkpoint inhibitors (ICIs). ${ }^{6}$ CheckMate032 was a phase I/II clinical trial to evaluate the efficacy and safety of nivolumab (anti-PD-1) plus ipilimumab (anti-CTLA4) on extensive-stage SCLC. ${ }^{7}$ In this trial, a total of 216 patients were enrolled and treated. An objective response rate was $10 \%-33 \%$ depending on the drug combinations and dosage. However, high rate of grade 3 or 4 treatment-related adverse events $(13 \%-30 \%$, depending on the dosage) was not satisfactory. Other clinical trials of ICIs include KeyNote-158 (ClinicalTrials.gov identifier: NCT02628067, a phase II trial investigating pembrolizumab in relapsed or refractory SCLC) $^{1}$ and IMpower133 that combined atezolizumab (anti-PD-L1) with carboplatin and etoposide. ${ }^{8}$ Overall, the clinical data on the efficacy of ICIs monotherapy in SCLC are not very promising. ${ }^{2}$ Therefore, seeking other forms of immunotherapy with more potency 
is desperately needed, for example, bispecific antibody and chimeric antigen receptor (CAR)-modified T cells.

Delta-like 3 (DLL3) is a single-pass type I transmembrane protein. The extracellular domain of DLL3 interacts with Notch receptor. ${ }^{9}$ A host of studies repeatedly and consistently indicated that DLL3 is an attractive target for cancer immunotherapy, particularly in SCLC and to a less extent in other cancer types. ${ }^{10-14}$ DLL3 is selectively expressing on the vast majority of SCLC ( 70\% in prevalence) with minimal or absent expression in normal adult tissues, even though the expression level might be magnitude lower than a typical immunotherapy target. $^{10} 1115$ DLL3-targeted antibody-drug conjugate (ADC) rovalpituzumab tesirine (Rova-T, SC16LD6.5) has shown durable tumor regression in vivo across multiple Patient-Derived Xenograft (PDX) models. ${ }^{16}$ Phase I clinical trial of Rova-T on SCLC presented some promising results in terms of efficacy, with $18 \%$ (11 out of 60 ) of treated patients having shown objective response, and $68 \%$ (41 out of 60 ) disease stabilization. ${ }^{17}$ However, the outcomes of the other following clinical trials were questionable or somewhat dampening. ${ }^{9}$ Nevertheless, the specificity of this target in SCLC encouraged us to seek the efficacy of DLL3-targeted T cell-based immunotherapy. As a proof of concept, we made a T cell-engaging bispecific antibody and CAR based on the mAb SC16.15. The cytotoxicity of DLL3-targeted bispecific antibody and CAR-T was tested in vitro and in xenograft mice.

\section{METHODS \\ Cell lines}

Three SCLC cell lines (H446, H196, H82) and DLL3negative A431 cell line were maintained as adherent monolayer cultures in Dulbecco's Modified Eagle Medium (DMEM) medium (Invitrogen, Carlsbad, California) supplemented with $10 \%$ fetal bovine serum (HyClone, Logan, Utah), 1\% L-glutamine, and 1\% penicillin-streptomycin (Invitrogen) and incubated in 5\% $\mathrm{CO}_{2}$ with a balance of air at $37^{\circ} \mathrm{C}$. Cells were harvested and the media were refreshed two times a week. DLL3negative A431 cells (human epithelial carcinoma cell line) were engineered to overexpress DLL3 by transfection with a plasmid encoding the full-length DLL3 (GeneBank accession number NP_058637.1). Both A431 and the stably transfected A431 (DLL3) cells were maintained in DMEM medium.

\section{Antibodies and western blot}

Antibody used in western blot for DLL3 was from Proteintech Group (Wuhan, China). Cells were cultured in T-25 or T-75 flasks for 3-5 days at a final confluence of $70 \%$. Cells were collected and lysed with lysis buffer ( $50 \mathrm{mM}$ Tris-HCl, pH 7.5, $50 \mathrm{mM} \mathrm{NaCl}, 5 \mathrm{mM}$ EDTA, $1 \%$ Triton X-100, mixed with cocktail proteinase inhibitors from Roche Applied Science (Indianapolis, Indiana)). Protein concentration of the cell lysate was measured by Coomassie Plus (Bradford) Protein Assay from Thermo
Scientific (Rockford, Illinois). Fifty micrograms of total protein were run on reducing sodium dodecyl sulfatepolyacrylamide gel electrophoresis (SDS-PAGE) for western blot analysis.

\section{Construction of DLL3 bispecific antibody}

The DLL3-targeted SC16.15 scFv (sequence from patent US9173959B1) was fused with hFc knob mutant (N297A/ S354C/T366W). By using an EcoRV/BspEI double digestion cloning method, the scFv coding sequence was cloned into the knob expression plasmid, placed downstream of a mouse IgG secretion signal peptide. The amino acid sequence of the knob cassette reads as: MEWSWVFLFFLSVTTGVHSDIQVQLQQSGAELAKPGASVKMSCKASGYT FTRYWIHWIKQRPGQGLEWIGYINPTTVYTEFNQNFKDKA TLTADKSSTTASMQLSSLTSEDSAVYYCARGGSNFFDYWG QGTTLTVSSGGGGSGGGGSGGGGSDIQMTQSPASLAASVG ETVAITCRASENIYYNLAWYQQKQGKSPQLLIYTANSLED GVPSRFSGSGSGTQYSLKINSMQPEDSATYFCKQAYDVPP TFGGGTKLEIKSGDKTHTCPPCPAPELLGGPSVFLFP PKPKDTLMISRTPEVTCVVVDVSHEDPEVKFNWYVD GVEVHNAKTKPREEQYASTYRVVSVLTVLHQDWLNGKE YKCKVSNKALPAPIEKTISKAKGQPREPQVYTLPPCREEM TKNQVSLWCLVKGFYPSDIAVEWESNGQPENNYKTT PPVLDSDGSFFLYSKLTVDKSRWQQGNVFSCSVMHE ALHNHYTQKSLSLSPGK (EcoRV/BspEI sites underlined, scFv in italic, and N297A/S354C/T366W mutations highlighted). The CD3-targeted OKT3 scFv was fused with $\mathrm{hFc}$ hole mutant (N297A/Y349C/T366S/L368A/ $\mathrm{Y} 407 \mathrm{~V})$. The OKT3 scFv coding sequence was cloned into the hole expression plasmid in a similar way. The amino acid sequence of the hole cassette reads as: MEWSWVFLFFLSVTTGVHSDIDIKLQQSGAELARPGASVKMSCKT SGYTFTRYTMHWVKQRPGQGLEWIGYINPSRGYTNYNQKF KDKATLTTDKSSSTAYMQLSSLTSEDSAVYYCARYYDDHY CLDYWGQGTTLTVSSVEGGSGGSGGSGGSGGVDDIQLTQS PAIMSASPGEKVTMTCRASSSVSYMNWYQQKSGTSPKRWI YDTSKVASGVPYRFSGSGSGTSYSLTISSMEAEDAATYYC QQWSSNPLTFGA GTKLELKSGDKTHTCPPCPAPELLG GPSVFLFPPKPKDTLMISRTPEVTCVVVDVSHEDPEVKFN WYVDGVEVHNAKTKPREEQYASTYRVVSVLTVLHQD WLNGKEYKCKVSNKALPAPIEKTISKAKGQPREPQV CTLPPSREEMTKNQVSLSCAVKGFYPSDIAVEWESN GQPENNYKTTPPVLDSDGSFFLVSKLTVDKSRWQQG NVFSCSVMHEALHNHYTQKSLSLSPGK (EcoRV/BspEI sites underlined, scFv in italic, and N297A/Y349C/T366S/ L368A/Y407V mutations highlighted). The knob and hole plasmids were mixed at 1:1 ratio and introduced into $293 \mathrm{~F}$ cells by polyethylenimine (PEI) transfection. The heterodimerized bispecific antibody was secreted into the medium and purified via protein A chromatography.

\section{Production of CAR-T cells}

The CAR expression cassette adopted the third generation format and was constructed by de novo gene synthesis, which consisted of the CD8a leader sequence (NP_001139345, a.a. 1-21), followed by anti-DLL3 $\mathrm{scFv}$ (SC16.15, sequence from patent US9173959B1) 
or isotype control HN3 (a human antibody that targets glypican-3, sequence from patent US20140044714), the CD8 $\alpha$ hinge (NP_001139345, a.a. 138-182), CD8 $\alpha$ transmembrane region (NP_001139345, a.a. 183-210), CD28 intracellular domain (NP_006130.1, a.a. 180-220), 4-1BB

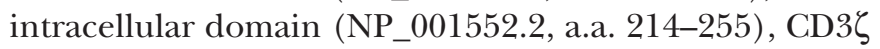
intracellular domain (NP_932170, a.a. 52-164), internal ribosome entry site (from encephalomyocarditis virus), and a red fluorescent protein mScarlet-I. The amino acid sequence of the CAR-HN3 cassette reads as: MALP VTALLLPLALLLHAARP DIQVQLVQSGGGLVQPGGSLRL SCAASYFDFDSYEMSWVRQAPGKGLEWIGSIYHSGSTYYN PSLKSRVTISRDNSKNTLYLQMNTLRAEDTATYYCARVNM DRFD YWGQGTLVTVSSSGTTTPAPRPPTPAPTIASQPL SLRPEACRPAAGGAVHTRGLDFACDIYIWAPLAGTC GVLLLSLVITLYCNHRNRSKRSRLLHSDYMNMTPRR PGPTRKHYQPYAPPRDFAAYRSKR GRKKLLYIFKQP FMRPVQTTQEEDGCSCRFPEEEEGGCELRVKFSRSA DAPAYQQGQNQLYNELNLGRREEYDVLDKRRGRDPE MGGKPQRRKNPQEGLYNELQKDKMAEAYSEIGMKGE RRRGKGHD GLYQGLSTATKD TYDALHMQALPPR (restriction enzyme sites underlined, HN3 in italic). CARSC16.15 had the same sequence except that HN3 part was replaced by SC16.15 coding sequence. Human peripheral blood mononuclear cells (PBMC) were isolated from the whole blood of healthy donors (Wuhan Blood Center) by a Ficoll separation method (Stem Cell Technologies, Vancouver, British Columbia, Canada). PBMC were cultured in RPMI 1640 medium supplemented with $200 \mathrm{IU} / \mathrm{mL}$ human recombinant interleukin (IL)-2 and activated by Dynabeads CD3/CD28 human T-activator (Cat. 11131D, ThermoFisher, Waltham, Massachusetts) for 3 days according to the manufacturer's instruction. The activated PBMC were transduced with lentivirus carrying the CAR cassette for 24hours. After transduction, refreshed medium containing $200 \mathrm{IU} / \mathrm{mL}$ of IL-2 was used to culture the CAR-T cells for additional 3 days before the functional studies. The expression of CAR genes was indicated by the expression of $\mathrm{mScarlet-I}$ protein as detected by flow cytometry.

\section{Flow cytometry method}

Cells were harvested by detaching with trypsin-EDTA (ThermoFisher, Waltham, Massachusetts), washed by centrifugation, and resuspended in ice-cold phosphate buffer saline (PBS) containing $5 \%$ bovine serum albumin (BSA). One million of cells per $\mathrm{mL}$ were incubated with $10 \mu \mathrm{g} / \mathrm{mL}$ of DLL3 bispecific antibody. The antibody binding was detected by phycoerythrin-conjugated goat antihuman IgG (Jackson ImmunoResearch, West Grove, Pennsylvania). The fluorescence associated with the live cells was measured using an FACS Calibur (BD Biosciences, Franklin Lakes, New Jersey). For PD-L1 expression analysis, a rabbit monoclonal antibody (Sinobiological, Beijing, China) was incubated with the SCLC cells and the antibody binding was detected by allophycocyanin (APC)conjugated goat-anti-rabbit IgG (Jackson ImmunoResearch, West Grove, Pennsylvania). For PD-1 expression analysis, a PD-1 inhibitory antibody (in-house made as scFv-hFc format, using nivolumab VH and VL sequences) was incubated with CAR-T cells and the antibody binding was detected by APC-conjugated goat-anti-human IgG (Jackson ImmunoResearch, West Grove, Pennsylvania).

\section{In vitro cytotoxicity assay}

In vitro cell killing activity of the bispecific antibody and CAR-T cells was assessed by firefly luciferase reporter assay. Cancer cells were stably transduced with a lentiviral vector that carries a luciferase reporter gene (ffLuc2). Two hundred microliters of stably transduced cells were seeded on a 96 -well plate $\left(10^{4}\right.$ cells/well $)$, with the addition of PBMC and bispecific antibody at the indicated concentrations, or addition of CAR-T cells at the indicated amount. Untreated cells were used as control. The cell mixtures were incubated at $37^{\circ} \mathrm{C}$ for 48 hours and then collected by spinning down. Collected cells were then lysed by two rounds of freezing-thawing. The released luciferase activity was measured to represent the cell viability. The in vitro cytotoxicity assay was repeated three times and PBMC from a total of three donors was tested. Reported was the representative result.

\section{In vivo efficacy study}

Five million H446 and A431 (DLL3) cells were subcutaneously injected into NOD/SCID/IL2Rg(null) (NSG) mice. After the tumor formed and reached the size of $100-200 \mathrm{~mm}^{3}$, the treatment was started by splenocyte depletion with $200 \mathrm{mg} / \mathrm{kg}$ cyclophosphamide (SigmaAldrich, St. Louis, Missouri). One day later, mice were intraperitoneally injected with 10 millions of PBMC cells or 5 millions of CAR-T cells, and intravenously injected with bispecific antibody and PD-1 antibody. The bispecific antibody was given two times at the indicated time point, and PD-1 antibody was given once every week. Tumor dimensions were determined every 2 or 3 days with a caliper. Tumor volume $\left(\mathrm{mm}^{3}\right)$ was calculated by the formula: $(a) \times\left(b^{2}\right) \times 0.5$, where $a$ is tumor length and $b$ is tumor width in millimeters. Five mice per group were assigned. The in vivo study was repeated two times with two different donors as the source of PBMC.

\section{Statistical analysis}

All statistical analyses were conducted using GraphPad Prism5 (GraphPad Software, La Jolla, California) and expressed as the mean \pm SEM. Comparison of two groups was performed using paired Student's t-test (two tailed). Comparisons among three or more groups were performed using one-way analysis of variance. $\mathrm{P}<0.05$ was considered statistically significant.

\section{RESULTS}

\section{Preparation of DLL3-targeted bispecific antibody}

We used the classical knob-into-hole structure to make the bispecific antibody. ${ }^{18}$ The anti-DLL3 scFv SC16.15 was fused with a human Fc knob and the anti-CD3 scFv 


\section{A}

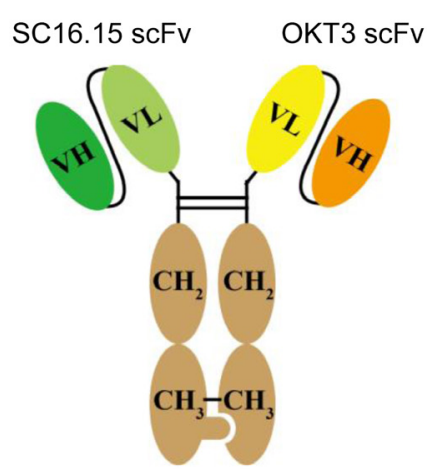

B

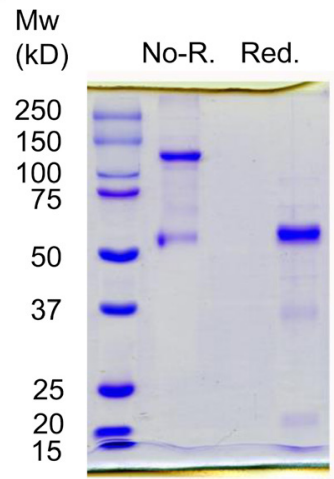

Figure 1 Preparation of delta-like 3 (DLL3) bispecific antibody. (A) Schematic diagram of the primary structure of the DLL3 bispecific antibody. The anti-DLL3 ScFv (SC16.15) was fused with $\mathrm{hFc}$ knob, and the anti-CD3 scFv (OKT3) was fused with hFc hole. (B) SDS-PAGE analysis of the purified bispecific antibody. Two micrograms of protein were loaded for each lane. Non-R., non-reduced condition, showing the dimerized bispecific antibody; Red., 2-mercaptoethanol reduced condition, showing the reduced monomer of the bispecific antibody.

OKT3 was fused with Fc hole (figure 1A). Both the knob and hole plasmid were coexpressed in $293 \mathrm{~F}$ cells. The heterodimerized bispecific antibody was purified via protein A affinity chromatography and the purity was accessed by SDS-PAGE (figure 1B). As expected, the nonreduced heterodimer migrated mainly as $120 \mathrm{kD}$ and the reduced monomers of both knob and hole migrated as about $60 \mathrm{kD}$.

\section{Cell binding specificity of the bispecific antibody}

Cell binding was checked on both DLL3-negative and DLL3-positive cancer cell lines, $\mathrm{T}$ lymphoma cell line Jurkat, and primary human T cells (PBMC; figure 2). Since DLL3 are generally expressed at a very low level on SCLC, ${ }^{10}$ as expected, the bispecific antibody marginally bound to SCLC cell line H446, H196, and H82 (figure 2A). To confirm the cell binding activity, we made an artificial A431 (DLL3) cell line by overexpressing DLL3 on A431 cells via lentiviral transduction and cell sorting. A little surprised, it was difficult to get DLL3 super high expressers (figure 2A, A431 (DLL3)), which probably explained why DLL3 is generally low expressing in SCLC cell lines and tissues. The binding of the bispecific antibody to $\mathrm{T}$ cells was apparent (figure 2B), as shown in both the Jurkat cell line and PBMC. To further confirm the DLL3 expression in the tested cell lines, we also ran western blot (figure 2C), which was consistent with the cell binding data.

\section{Cytotoxicity of DLL3 bispecific antibody}

Cytotoxicity of the DLL3 bispecific antibody was tested in DLL3-negative A431 cells and DLL3-positive SCLC cell lines. All the tested cell lines were stably transduced with a lentiviral vector to express the firefly luciferase gene that was utilized to measure the cytotoxicity of the bispecific

A
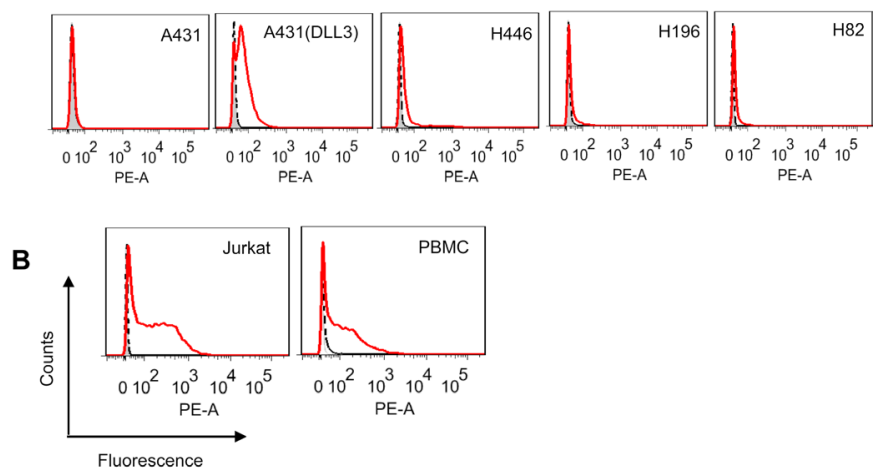

C

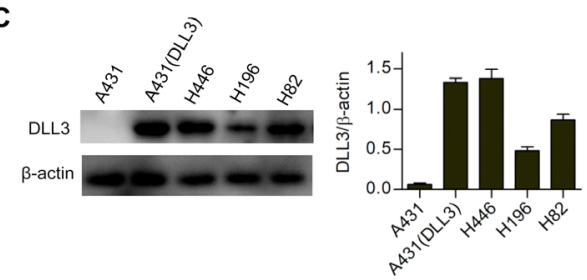

Figure 2 Binding properties of the delta-like 3 (DLL3) bispecific antibody. (A) Flow cytometry analysis of the bispecific antibody binding to different cancer cell lines. Ten micrograms of the bispecific antibody were coincubated with one million of cells. Antibody binding was detected by phycoerythrin-conjugated goat antihuman IgG. Shaded area, secondary antibody staining; dashed lines, isotype control (pooled human IgG) staining; red solid line, bispecific antibody staining. (B) T cell binding analysis of the bispecific antibody. Same experimental settings were used as above mentioned, except that the $\mathrm{T}$ cell line Jurkat and peripheral blood mononuclear cells were tested. (C) Western blot analysis of the DLL3 expression in different cancer cell lines. Fifty micrograms of total protein from each cell lysate were run on reduced SDS-PAGE, followed by anti-DLL3 antibody staining. The $\beta$-actin was used loading control. A431 (DLL3) was an artificial cell line that was forcefully overexpressing DLL3. The band intensity from each lane was quantified by using ImageJ software, and presented as mean \pm SEM.

antibody. As shown in figure 3, the bispecific antibody efficiently killed the DLL3-positive H446 and H196 cells in a dose-dependent manner in the presence of unstimulated PBMC, while had little influence on the A431 cells. The artificial A431 (DLL3) and H82 cells were relatively resistant to the bispecific antibody-mediated T cell killing.

\section{Tumor-suppression activity of DLL3 bispecific antibody in xenograft mice}

The efficient in vitro killing result of the bispecific antibody encouraged us to investigate its in vivo efficacy. Because the classical PD-L1/PD-1 immune checkpoint plays significant roles in limiting the efficacy of $\mathrm{T}$ cell immunotherapy in many cancer types, and SCLC cells have generally low-level expression of PD-L1 $1{ }^{19}$ we decided to include PD-1 blockade as a combination therapy with the DLL3 bispecific antibody for the in vivo study. First, we confirmed the PD-L1 expression on H446 and A431 (DLL3) cells by flow cytometry (figure 4A), and the PD-1 


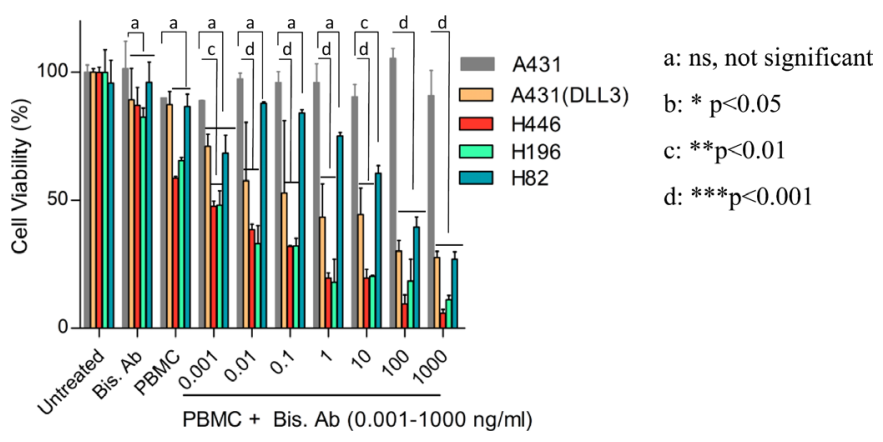

Figure 3 In vitro cell killing assay of the delta-like 3 (DLL3) bispecific antibody. All the tested cell lines were stably transduced to constitutively express a fire fly luciferase reporter gene (ffLuc2). Ten thousand cancer cells were coincubated with two hundred thousand unstimulated peripheral blood mononuclear cells (PBMC) cells for 48 hours in the presence of variable concentrations of the bispecific antibody as indicated. The cell viability was quantified by measuring the intracellular luciferase activity. DLL3-negative A431 was served as a control of the non-specific killing by the bispecific antibody. Statistical comparisons between the A431 control and other cell lines at each bispecific antibody concentration was calculated and labeled on the top of the bar. Data represent mean \pm SEM.

expression on the DLL3 bispecific antibody activated PBMC (figure 4B). Then, NSG mice were subcutaneously inoculated with H446 and A431 (DLL3) cells. When the tumor reached the size of $100-200 \mathrm{~mm}^{3}$, treatment was started by intraperitoneal injection of 10 million PBMC and tail vein injection of $1.0 \mathrm{or} 0.5 \mathrm{mg} / \mathrm{kg}$ bispecific antibody. As shown in figure 4, the bispecific antibody significantly suppressed tumor growth in both H446 and A431 (DLL3) cells (figure 4C), with better efficacy in A431 (DLL3) than H446, which was in contrast with the in vitro killing data that the bispecific antibody had better killing on H446 than A431 (DLL3). Addition of the PD-1 inhibitory antibody (in-house made as scFv-hFc format, using nivolumab VH and VL sequences) dramatically enhanced the antitumor activity of the bispecific antibody and prolonged the survival of both H446 and A431 (DLL3; figure 4E), indicating that combination therapy of DLL3 bispecific antibody with PD-1 inhibition may potentially maximize the benefit of immunotherapy in SCLC. The loss of the body weight in the treated mice was apparent and associated with the dosage of the bispecific antibody (figure 4G).

\section{Cytotoxicity of DLL3-targeted CAR-T cells}

As we have already seen the cytotoxicity and in vivo efficacy of the bispecific antibody, it would be interesting to see the potency of the DLL3-targeted CAR-T cells. As shown in figure 5, the CAR was constructed by de novo gene synthesis (figure 5A), packed into a lentiviral vector, and transduced into PBMC. The transduction efficiency was indicated by the parentage of the mScarlet-I positive population (figure 5B), which was about $50 \%$ in both DLL3 and HN3 control CAR-T group. HN3 was a VH-domain antibody that targets glypican-3, a cell surface biomarker that is mainly expressing on hepatocellular carcinoma. ${ }^{20}$ The in vitro killing of DLL3 CAR-T cells was apparent, especially on native SCLC cells H446 and H196 (figure 5C). The maximal killing was seen at 10:1 ratio (CAR-T: tumor cells) for A431 (DLL3) and H446, and 5:1 for H196. No maximal killing was achieved for $\mathrm{H} 82$ at up to $15: 1$ ratio.

\section{In vivo efficacy of DLL3 CAR-T cells}

The in vivo efficacy was tested on H446 model. Based on the same rationale as in the bispecific antibody combination treatment, we also included PD-1 blockade as a combination in the CAR-T treatment. As shown in figure 6A, both the DLL3 CAR-T and the HN3 control CAR-T cells expressed PD-1 as examined by flow cytometry. The in vivo study showed that the DLL3 CAR-T significantly slowed down the tumor growth, but surprisingly, addition of PD-1 inhibitory antibody did not enhance the tumor growth suppression (figure 6B) or prolong the survival of the mice (figure 6C), which was not the case for the bispecific antibody combination treatment (figure 4C). The loss of body weight in the CAR-T treatment group was also apparent (figure 6D). Taken together, these data suggested that combination of DLL3-targeted bispecific antibody and PD-1 blockade was superior to CAR-T combination.

\section{DISCUSSION}

SCLC is a highly aggressive disease that novel curative therapies are imperative, given that the overall prognosis remains poor. Conventional chemotherapy plus radiotherapy are not very effective due to the rapid onset of relapse and drug resistance, and the survival rate of patient with SCLC has not been improved over the past three decades. ${ }^{21}$ In this disappointing scenario, there is a strong rationale to test immunotherapy that has changed the paradigm of treatment of some cancer types, especially circulating tumors. So far, ICI antibodies and DLL3targeted ADC are the two front-running forms of SCLC immunotherapy. ${ }^{6} 1522$ In the current study, we aimed to explore the therapeutic potential of the third form of immunotherapy, bispecific antibody and CAR-T, both of which are based on the mobilization/reactivation of $\mathrm{T}$ cells.

We used the classical knob-into-hole structure to make the bispecific antibody in the heterodimerized scFv-hFc format. DLL3-specific and CD3-specific scFv was utilized as the targeting moieties. Cell binding and cytotoxicity assays showed that the bispecific antibody was able to kill DLL3-positive cancer cells efficiently in the working concentration of $1-1000 \mathrm{ng} / \mathrm{mL}$ (figure 3), even though the cell binding of the bispecific antibody was very weak (figure 2A). Previous studies demonstrated that DLL3 is remarkably low-abundance protein on the surface of tumor cells, in the order of only 10000 molecules per cell, and H82 (the same cell line used in the current study) is representative of median DLL3 expression, with 14000 
A

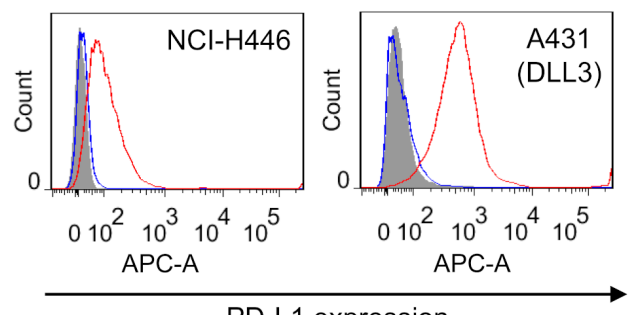

PD-L1 expression
B

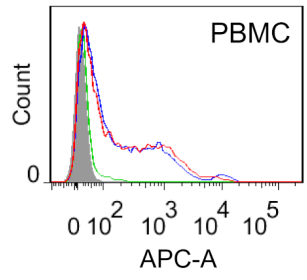

PD-1 expression
C $\mathrm{NCl}-\mathrm{H} 446$

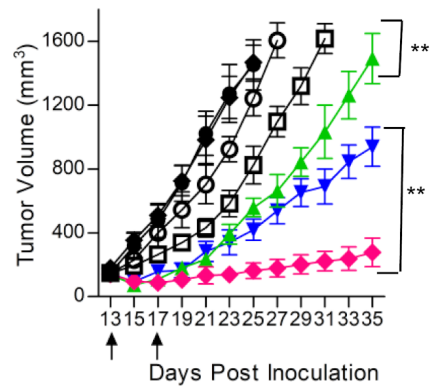

E NCl-H446

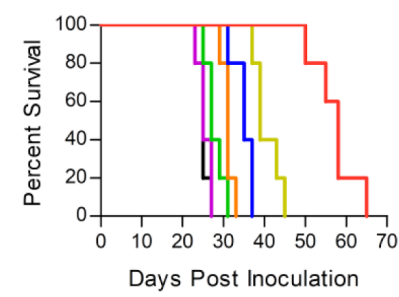

G NCl-H446

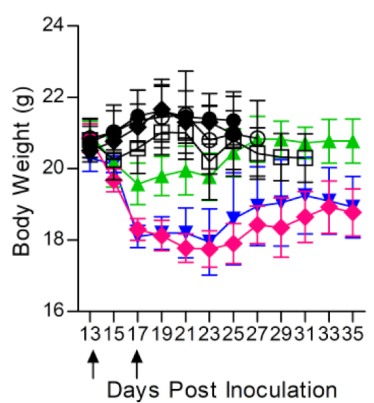

D A431(DLL3)

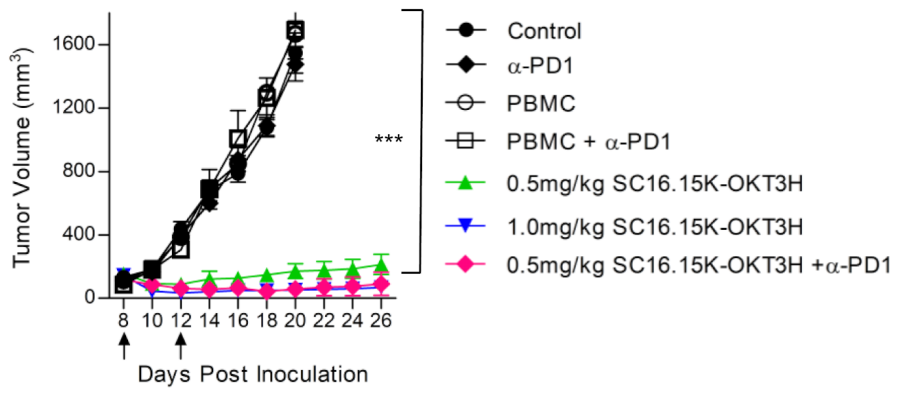

\section{F A431(DLL3)}

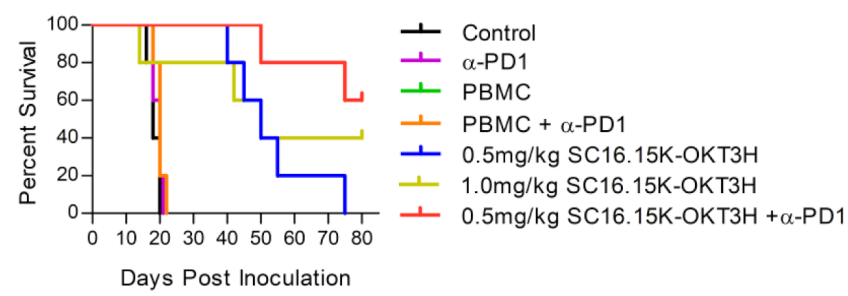

\section{H A431(DLL3)}

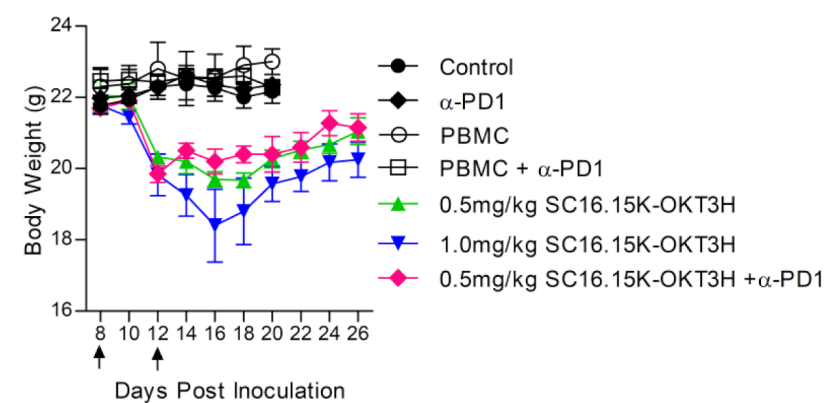

Figure 4 In vivo efficacy testing of the delta-like 3 (DLL3) bispecific antibody. (A) PD-L1 expression on H446 and A431 (DLL3) cells. One million of cells was stained with PD-L1 rabbit monoclonal antibody, followed by detection with APC-conjugated goatanti-rabbit IgG. Shaded area, secondary antibody staining; blue curve, isotype control (pooled rabbit lgG) staining; red curve, PD-L1 antibody staining. (B) PD-1 expression on the DLL3 bispecific antibody-stimulated peripheral blood mononuclear cells (PBMC) cells. H446 (red curve) and A431(DLL3) cells (blue curve) were incubated with PBMC for 48 hours in the presence of $100 \mathrm{ng} / \mathrm{mL}$ DLL3 bispecific antibody, followed by flow cytometry to analyze PD-1 expression. Shaded area, unstimulated PBMC cell staining; green curve, isotype control (pooled human IgG) staining of H446-stimulated PBMC. (C) Tumor growth curve of native small cell lung cancer cell line NCl-H446. Five million cells were subcutaneously inoculated in each NSG mouse. After the tumor formed and reached a size of $100-200 \mathrm{~mm}^{3}$, treatment was started. Bispecific antibody alone $(0.5 \mathrm{mg} / \mathrm{kg}$ or $1.0 \mathrm{mg} /$ $\mathrm{kg}$ body weight), or in combination of anti-PD-1 antibody (in-house made scFv-hFc format, $5.0 \mathrm{mg} / \mathrm{kg}$ ) were tested. Ten million unstimulated human PBMC were intraperitoneally given immediately before the first intravenous delivery of the antibody. Arrows indicated the injection time point of the bispecific antibody. The PD-1 antibody was intravenously given once every week since the start of the treatment. Both tumor volume and body weight were measured every two or 3 days. ${ }^{* *} p<0.01$, calculated using a Student's paired t-test (two-tailed), ${ }^{* * *} p<0.001$. (D) Tumor growth curve of A431 (DLL3), an artificial A431 cell line that was forcefully overexpressing DLL3. ${ }^{* * *} p<0.001$, calculated using a Student's paired t-test (two tailed). (E) The survival curves of the H446 mouse model treated with the bispecific antibody. (F) The survival curves of the A431 (DLL3) mouse model. (G) Body weight of the H446 mouse model treated with the bispecific antibody. (H) Body weight of the A431 (DLL3) mouse model. 
A

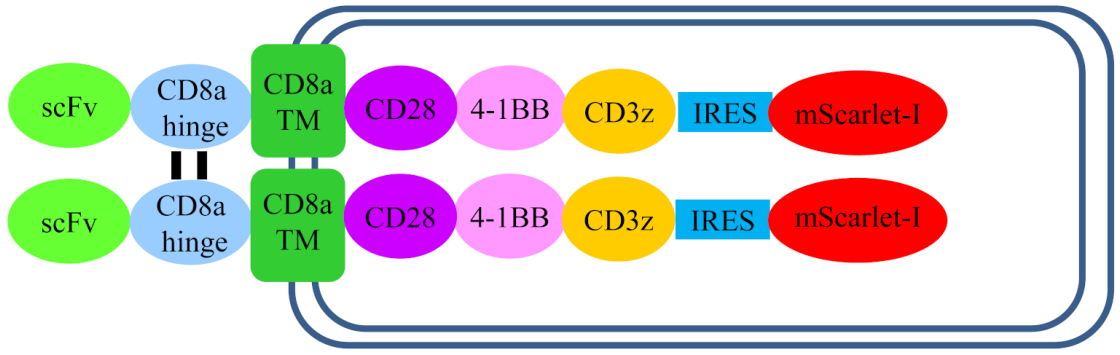

B
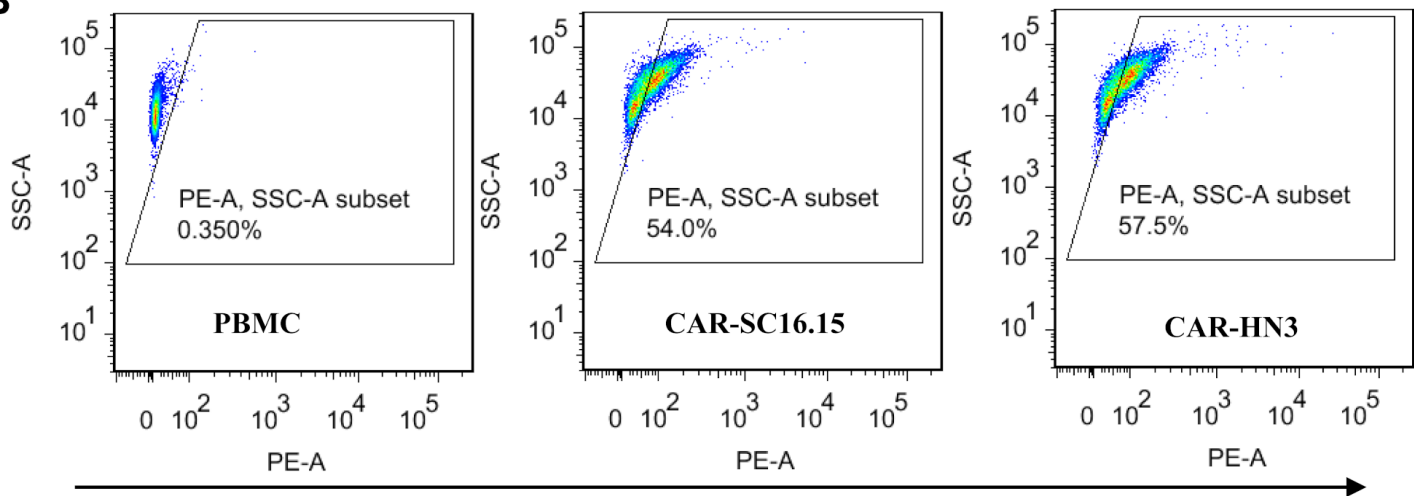

mScarlet-I fluorescence intensity

C

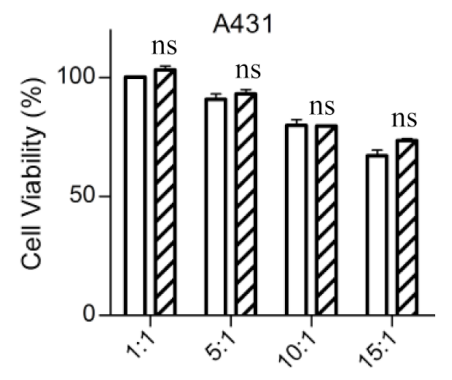

CAR-T:tumor cell ratio

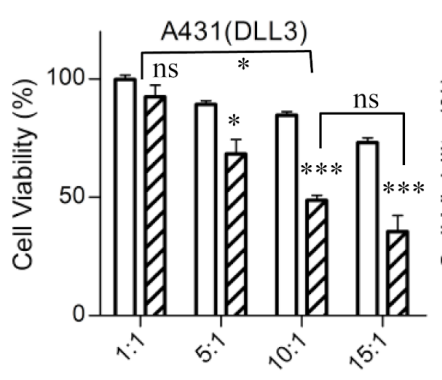

CAR-T:tumor cell ratio

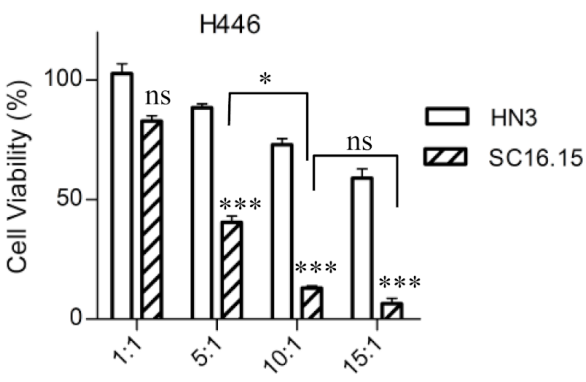

CAR-T:tumor cell ratio

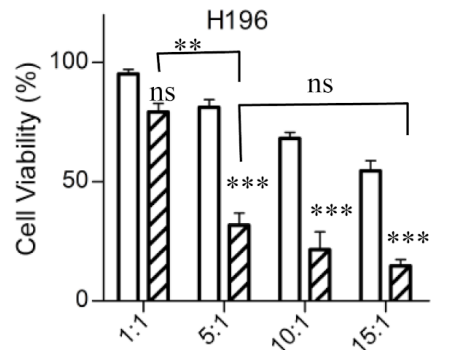

CAR-T:tumor cell ratio

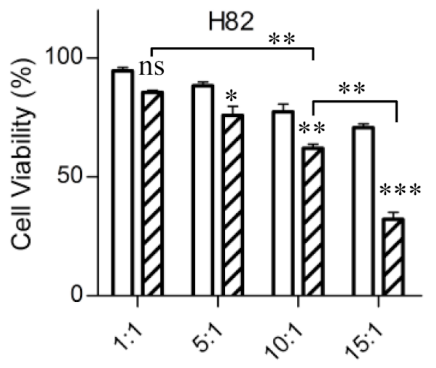

CAR-T:tumor cell ratio

Figure 5 In vitro cell killing assay of the delta-like 3 (DLL3)-targeted chimeric antigen receptor (CAR)-T. (A) Schematic diagram of the CAR structure. Anti-DLL3 scFv (SC16.15) or isotype control (HN3, a human antibody that targets glypican-3, sequence from patent US20140044714) was fused with the following domains, the CD8 $\alpha$ hinge, CD8 $\alpha$ transmembrane region, CD28 intracellular domain, 4-1BB intracellular domain, CD3 $\zeta$ intracellular domain, internal ribosome entry site (IRES, from encephalomyocarditis virus), and a red fluorescent protein mScarlet-I. (B) Transduction efficiency analysis of the CAR-T cells, as indicated by the expression of mScarlet-I. Untransduced PBMC was used as negative control. (C) Cell killing measurement of the CAR-T cells. All the cancer cell lines were stably transduced to constitutively express a fire fly luciferase reporter gene (ffLuc2). Ten thousand cancer cells were coincubated with different amount of CAR-T cells for 48 hours. The cell viability was quantified by measuring the intracellular luciferase activity. HN3 was a negative control CAR that targets glypican-3. Data represent mean \pm SEM. Statistical comparisons between the DLL3 CAR-T (SC16.15) and the control (HN3) at each CAR-T: tumor cell ratio was calculated and labeled on the top of the bar. Comparisons between different DLL3 CAR-T ratios were also calculated. ns, not significant, ${ }^{*} p<0.05,{ }^{* \star} p<0.01,{ }^{* \star *} p<0.001$. 
A

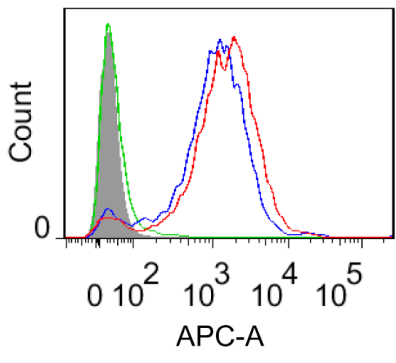

PD-1 expression
B

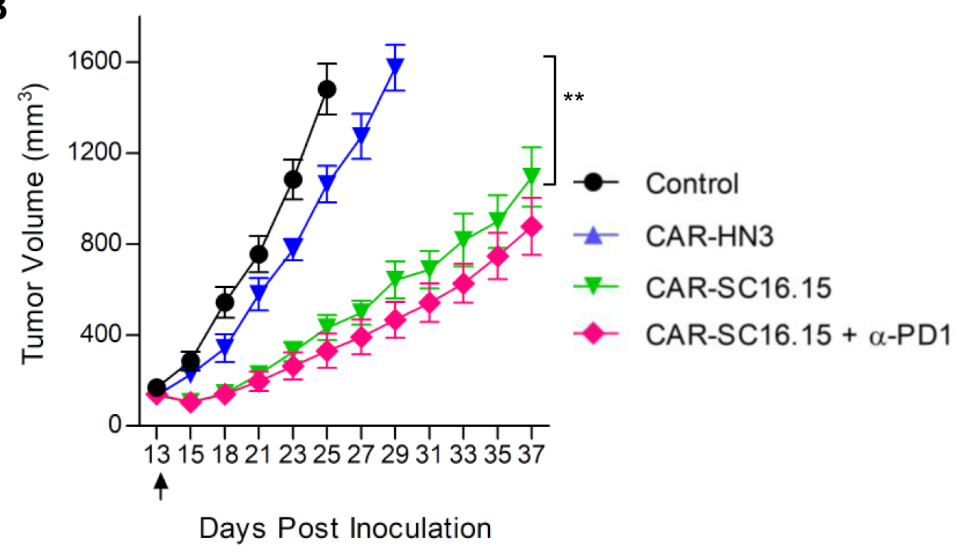

C

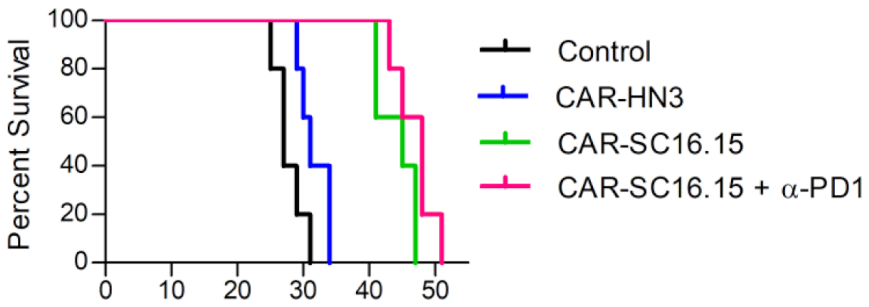

Days Post Inoculation

D

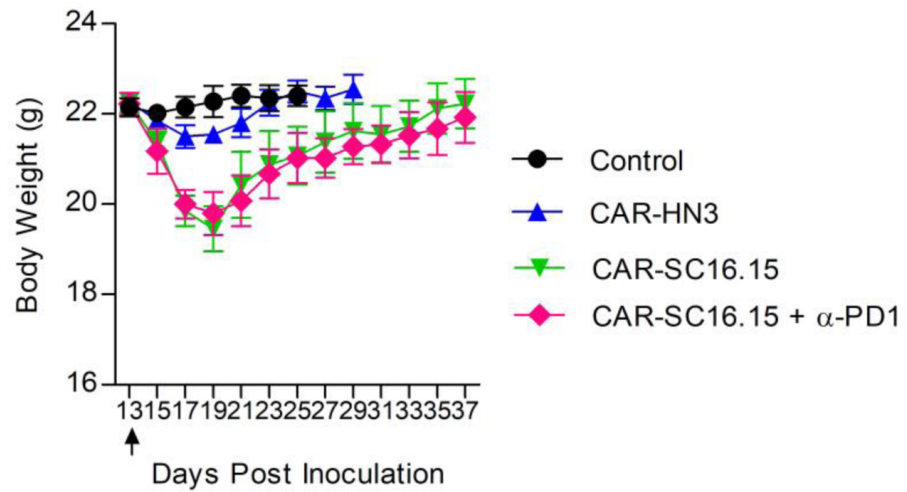

Figure 6 In vivo efficacy testing of the delta-like 3 (DLL3)-targeted chimeric antigen receptor (CAR)-T cells. (A) PD-1 expression on CAR-T cells. DLL3 CAR-T cells (red curve) and HN3 control CAR-T cells (blue curve) were stained with the PD-1 inhibitory antibody (in-house made scFv-hFc format), followed by APC-conjugated goat-anti-human IgG. Shaded area, peripheral blood mononuclear cells cell staining; green curve, isotype control (pooled human IgG) staining of the DLL3 CAR-T cells. (B) Tumor growth curve of NCl-H446. Five million cells were subcutaneously inoculated in NSG mice. After the tumor formed and reached a size of $100-200 \mathrm{~mm}^{3}$, treatment was started by intraperitoneal delivery of five million CAR-T cells. Arrow indicated the time point of the CAR-T treatment. The anti-PD-1 antibody (in-house made scFv-hFc format, $5.0 \mathrm{mg} / \mathrm{kg}$ ) was intravenously given once every week since the start of the treatment. Both tumor volume and body weight were measured every 2 or 3 days. ${ }^{\star *} p<0.01$, calculated by using a paired Student's t-test. (C) The survival curves of the treated mice. (D) The body weight of the treated mice.

molecules per cell. ${ }^{10}$ The low expression level of DLL3 explains the weak binding of the bispecific antibody in the current study. We would optimistically postulate that a bispecific antibody with higher affinity for DLL3 might compensate for the drawback of low-level expression of the target and achieve better efficacy. Another study demonstrated that DLL3-targeted ADC rovalpituzumab tesirine was potent in the SCLC and large cell neuroendocrine carcinoma PDX models, and durable regression of tumor growth was achieved. ${ }^{16}$ It would be interesting to compare the ADC and bispecific antibody under the same experimental settings, for example, the same tested cell lines and mouse models.

CAR-T has a similar mode of action as bispecific antibody, both of which is able to activate T cells to kill cancer cells. However, the way of the T cell activation in CAR-T and bispecific antibody is different. The intracellular costimulation domains in the CAR can directly activate $\mathrm{T}$ cell without the need of tumor antigen association, while bispecific antibody stimulates $\mathrm{T}$ cells in a way more like how the T cells are naturally activated, for example, tumor antigen binding-triggered clustering of the CD3 complex 
in the $\mathrm{T}$ cells, which delivers the cross-membrane activation signals in $\mathrm{T}$ cells. In the current study, it was found that the potency of the DLL3-targeted CAR-T cells was similar as the bispecific antibody. However, the CAR-T cells could not be potentiated by the inclusion of PD-1 inhibition, which was in contrast with that of the bispecific antibody combination, suggesting that the fate of $\mathrm{T}$ cells activated by bispecific antibody differs substantially from that by CAR. A similar observation was also found that the GD2-targeted bispecific antibody had superior activity than CAR-T in a melanoma xenograft mouse model, because the bispecific antibody did not induce T-cell death mediated by CAR. ${ }^{23}$ In the same study, the authors found that majority of high CAR density T cells were depleted on exposure to target cells while the bispecific antibody redirected $\mathrm{T}$ cells survived, and blockade of PD-1 did not prevent CAR-T cell depletion. Another interesting study showed that long-lasting PD-1 blockade in CAR-T cells by shRNA actually impaired $\mathrm{T}$ cell antitumor function, mainly because long-lasting PD-1 blockade inhibited $\mathrm{T}$ cell proliferation and prevented effector $\mathrm{T}$ cells from differentiating into effect memory $\mathrm{T}$ cells. ${ }^{24}$ Clinical experience employing the combination of CAR-T and immune checkpoint blockade is in its early stages, and there are both encouraging preliminary results and some unsatisfactory outcomes. ${ }^{25} \mathrm{~A}$ small phase I clinical trial in a GD2-CAR for relapsed or refractory neuroblastoma did not find significant benefit of combining PD-1 blockade. ${ }^{26}$

It should be noted that the epitopes of the bispecific antibody may have pivotal impacts on the activity. ${ }^{18} 27$ Bispecific antibodies that target epitopes closer to the cell membrane are generally advantageous as this may facilitate to form a stronger immune synapse between $\mathrm{T}$ cells and cancer cells. ${ }^{27}$ It is unclear whether the epitope of SC16.15 is the best fit for DLL3-targeted bispecific antibody. Therefore, following-up studies are needed to test more bispecifics that target different epitopes of DLL3 to achieve the optimal therapeutic benefit of DLL3-targeted immunotherapy.

\section{CONCLUSION}

The current study demonstrated that both DLL3-targeted bispecific antibody and CAR-T cells were able to selectively kill DLL3-positive cancer cells in vitro and suppress tumor growth in vivo. Combination of PD-1 blockade was capable of enhancing the activity of bispecific antibody but not the CAR-T cells, suggesting its superiority over CAR-T in terms of efficacy. Further studies to optimize the DLL3 bispecifics are worthwhile to fully take the advantage of DLL3-targeted immunotherapy.

Acknowledgements The authors would like to thank Dr. Shaozhong Wei, president of Hubei Cancer Hospital, for discussing the project and for reviewing the manuscript.

Contributors $X C$ performed the experiments, analyzed the data, prepared the figures, and drafted the manuscript. NA, YZ, and CX carried out the flow cytometry. $\mathrm{CW}, \mathrm{XY}$, and DW provided assistance with experiments and data analysis. MF designed the project and finalized the manuscript. All authors read and approved the final manuscript.

Funding This work was supported by the National Natural Science Foundation of China (31670943), the Fundamental Research Funds for the Central Universities (2662016PY113, 2662017PY111, and 2662019YJ013), the Applied Basic Research Program of Wuhan Science and Technology Bureau (2017060201010195), Bethune Medical Program of Jilin Provincial Science and Technology Department (20160101125JC).

Competing interests None declared.

Patient consent for publication Not required.

Ethics approval All the procedures used in the animal studies were approved by the Animal Care and Use Committee of Huazhong Agricultural University.

Provenance and peer review Not commissioned; externally peer reviewed.

Data availability statement Data sharing not applicable as no datasets generated and/or analyzed for this study. Data sharing not applicable as no datasets generated and/or analyzed for this study.

Open access This is an open access article distributed in accordance with the Creative Commons Attribution Non Commercial (CC BY-NC 4.0) license, which permits others to distribute, remix, adapt, build upon this work non-commercially, and license their derivative works on different terms, provided the original work is properly cited, appropriate credit is given, any changes made indicated, and the use is non-commercial. See http://creativecommons.org/licenses/by-nc/4.0/.

\section{ORCID iDs}

Norhan Amar http://orcid.org/0000-0002-9921-824X

Mingqian Feng http://orcid.org/0000-0002-0654-8224

\section{REFERENCES}

1 Wang S, Zimmermann S, Parikh K, et al. Current diagnosis and management of small-cell lung cancer. Mayo Clin Proc 2019;94:1599-622.

2 Pavan A, Attili I, Pasello G, et al. Immunotherapy in small-cell lung cancer: from molecular promises to clinical challenges. J Immunother Cancer 2019;7:205.

3 Povsic M, Enstone A, Wyn R, et al. Real-world effectiveness and tolerability of small-cell lung cancer (SCLC) treatments: a systematic literature review (SLR). PLoS One 2019;14:e0219622.

4 Micke P, Faldum A, Metz T, et al. Staging small cell lung cancer: Veterans Administration Lung Study Group versus International Association for the Study of Lung Cancer--what limits limited disease? Lung Cancer 2002;37:271-6.

5 Waqar SN, Morgensztern D. Treatment advances in small cell lung cancer (SCLC). Pharmacol Ther 2017;180:16-23.

6 Regzedmaa O, Zhang H, Liu H, et al. Immune checkpoint inhibitors for small cell lung cancer: opportunities and challenges. Onco Targets Ther 2019;12:4605-20.

7 Antonia SJ, López-Martin JA, Bendell J, et al. Nivolumab alone and nivolumab plus ipilimumab in recurrent small-cell lung cancer (CheckMate 032): a multicentre, open-label, phase 1/2 trial. Lancet Oncol 2016;17:883-95.

8 Horn L, Mansfield AS, Szczęsna A, et al. First-Line Atezolizumab plus chemotherapy in extensive-stage small-cell lung cancer. $N$ Engl $J$ Med 2018;379:2220-9.

9 Leonetti A, Facchinetti F, Minari R, et al. Notch pathway in small-cell lung cancer: from preclinical evidence to therapeutic challenges. Cell Oncol 2019;42:261-73.

10 Sharma SK, Pourat J, Abdel-Atti D, et al. Noninvasive interrogation of DLL3 expression in metastatic small cell lung cancer. Cancer Res 2017;77:3931-41.

11 Tanaka K, Isse K, Fujihira T, et al. Prevalence of delta-like protein 3 expression in patients with small cell lung cancer. Lung Cancer 2018;115:116-20.

12 Koshkin VS, Garcia JA, Reynolds J, et al. Transcriptomic and protein analysis of small-cell bladder cancer (SCBC) identifies prognostic biomarkers and DLL3 as a relevant therapeutic target. Clin Cancer Res 2019;25:210-21.

13 Puca L, Gavyert K, Sailer V, et al. Delta-like protein 3 expression and therapeutic targeting in neuroendocrine prostate cancer. Sci Transl Med 2019;11. doi:10.1126/scitranslmed.aav0891. [Epub ahead of print: 20 Mar 2019].

14 Spino M, Kurz SC, Chiriboga L, et al. Cell surface Notch ligand DLL3 is a therapeutic target in isocitrate Dehydrogenase-mutant glioma. Clin Cancer Res 2019;25:1261-71. 
15 Saito M, Saito K, Shiraishi K, et al. Identification of candidate responders for anti-PD-L1/PD-1 immunotherapy, Rova-T therapy, or $\mathrm{EZH} 2$ inhibitory therapy in small-cell lung cancer. Mol Clin Oncol 2018;8:310-4.

16 Saunders LR, Bankovich AJ, Anderson WC, et al. A DLL3-targeted antibody-drug conjugate eradicates high-grade pulmonary neuroendocrine tumor-initiating cells in vivo. Sci Trans/ Med 2015;7:302ra136.

17 Rudin CM, Pietanza MC, Bauer TM, et al. Rovalpituzumab tesirine, a DLL3-targeted antibody-drug conjugate, in recurrent small-cell lung cancer: a first-in-human, first-in-class, open-label, phase 1 study. Lancet Oncol 2017;18:42-51.

18 Qi J, Li X, Peng H, et al. Potent and selective antitumor activity of a T cell-engaging bispecific antibody targeting a membrane-proximal epitope of ROR1. Proc Natl Acad Sci U S A 2018;115:E5467-76.

19 Carbotti G, Nikpoor AR, Vacca P, et al. IL-27 mediates HLA class I up-regulation, which can be inhibited by the IL-6 pathway, in HLA-deficient small cell lung cancer cells. J Exp Clin Cancer Res 2017;36:140.

20 Feng M, Gao W, Wang R, et al. Therapeutically targeting glypican-3 via a conformation-specific single-domain antibody in hepatocellular carcinoma. Proc Natl Acad Sci U S A 2013;110:E1083-91.
21 Van Den Borg R, Leonetti A, Tiseo M, et al. Novel targeted strategies to overcome resistance in small-cell lung cancer: focus on PARP inhibitors and rovalpituzumab tesirine. Expert Rev Anticancer Ther 2019;19:461-71.

22 Lashari BH, Vallatharasu Y, Kolandra L, et al. Rovalpituzumab Tesirine: a novel DLL3-Targeting antibody-drug conjugate. Drugs $R D$ 2018;18:255-8.

23 Hoseini SS, Dobrenkov K, Pankov D, et al. Bispecific antibody does not induce T-cell death mediated by chimeric antigen receptor against disialoganglioside GD2. Oncoimmunology 2017;6:e1320625.

24 Wei J, Luo C, Wang Y, et al. PD-1 silencing impairs the anti-tumor function of chimeric antigen receptor modified $T$ cells by inhibiting proliferation activity. J Immunother Cancer 2019;7:209.

25 Grosser R, Cherkassky L, Chintala N, et al. Combination immunotherapy with CAR T cells and checkpoint blockade for the treatment of solid tumors. Cancer Cell 2019;36:471-82.

26 Heczey A, Louis CU, Savoldo B, et al. CAR T cells administered in combination with Lymphodepletion and PD-1 inhibition to patients with neuroblastoma. Mol Ther 2017;25:2214-24.

27 Li J, Stagg NJ, Johnston J, et al. Membrane-Proximal epitope facilitates efficient $\mathrm{T}$ cell synapse formation by Anti-FcRH5/ CD3 and is a requirement for myeloma cell killing. Cancer Cell 2017;31:383-95. 